\title{
EFFECT OF THE COMPLEXITY OF SUNFLOWER GROWING REGIONS ON THE GENETIC PROGRESS ACHIEVED BY BREEDING PROGRAMS
}

de la Vega, A.J. ${ }^{*}$

Pioneer Hi-Bred Services Co. Ltd., H-2040 Budaörs, Neumann J. u. 1, Hungary

Received: November 06, 2012

Accepted: December 01, 2012

\section{SUMMARY}

Sunflower (Helianthus annuus L.) breeding programs typically target heterogeneous regions, where large genotype $\times$ environment interactions (GEI) complicate genetic progress. Some understanding of the underlying factors, nature and repeatability of GEIs can help to accommodate their effects. This review summarizes the findings of a series of studies conducted in Argentina with the goals of understanding the effect of GEIs on sunflower yield progress and design breeding strategies aimed at impacting on future rates of genetic advance. Pattern analyses revealed that the central and northern subregions are different mega-environments (ME), which suggests that yield gains in the northern $\mathrm{ME}$ would have been reduced if selection had been done in central environments and vice versa. Photoperiod and minimum temperature during the reproductive phases are the main environmental factors underlying the observed GEIs. Genetic gain analyses indicated clear and continuous improvements in oil yield due to plant breeding during 25 years and confirmed the prediction about lack of yield gains under the northern $\mathrm{ME}$ from selection done in central environments. The central ME showed the highest and most consistent genetic progress, probably because it concentrates most breeding programs and shows the highest repeatability. Breeding progressed to merge the best combinations of two original hybrid groups, reducing the original maturity range within MEs and concentrating on different maturity types among MEs. The model of correlated response to selection indicated that dividing the central-north region of Argentina in central and northern MEs and exploiting specific adaptation would result in faster genetic progress. In the central ME, there is scope to replace years with locations at no costs in heritability. In the northern subregion, trials should be conducted over at least four years. Late planting dates in a central location associated positively with the northern environments in terms of genotypic discrimination; which constitutes an opportunity for indirect selection for the northern ME. Physiological models served to establish the importance of attributes and processes affecting crop performance in the post-anthesis phase in determining the observed genotypeby-planting time interaction. The diameter of the empty center and canopy stay green were found to be efficient secondary indicators related to seed set and

* Corresponding author: e-mail: abelardo.delavega@pioneer.com 
duration of grain filling, respectively - indicators that can be used to improve the efficiency of selection for specific adaptation to late plantings and to the northern ME.

Key words: adaptation analysis, genetic gain, genotype $\times$ environment interaction, Helianthus annuus $\mathrm{L}$., indirect selection, sunflower

\section{INTRODUCTION}

Sunflower is grown over a widely scattered geographical area that includes tropical, subtropical and temperate climates, different crop management systems and a broad and variable range of abiotic and biotic challenges. The sustainability of this production system largely relies on the ability of the breeding programs to increase the current rates of genetic progress for grain and oil yield in the different growing regions. Due to the relatively small value of the global sunflower seed market, few existing breeding programs aim to develop hybrids for large and heterogeneous target populations of environments (TPE; Comstock, 1977) where environmental variation strongly interacts with plant genotype, often giving rise to complex genotype $\times$ environment interactions (GEI) for yield (de la Vega and Chapman, 2010).

Genotype $\times$ environment interactions are detected as significant changes in the relative performance of the genotypes when they are evaluated in different environments. In complex TPEs, large and regional GEIs become a major challenge to genetic progress, since they complicate selection for broad adaptation. The analysis of plant adaptation, herein assumed to be any investigation of differences in performance of genotypes that is conducted to assist the achievements of breeding objectives, can help to accommodate their effects through the design and implementation of appropriate selection strategies (Cooper, 1999).

Adaptation analyses have been conducted for different crop growing systems, but they are scarce in the sunflower scientific literature, particularly for the main production areas. This review presents the conclusions of a series of sunflower studies conducted in Argentina with the aim of:

1. understanding the magnitude, repeatability and predictability of the observed GEIs;

2. estimating their effects on the past genetic progress for oil yield; and

3. formulating selection strategies aimed at increasing the future rate of genetic improvement.

Argentina constitutes a unique case study, since sunflower is grown on a large environmental variability across a $1500 \mathrm{~km}$ latitudinal range, from $26^{\circ} \mathrm{S}$ (Chaco; subtropical) to $39^{\circ} \mathrm{S}$ (southern Buenos Aires; temperate). Although sunflower is cultivated in Argentina in three subregions, i.e., northern, central and south, most of the cited studies were only focused on the first two. 


\section{Genotype $\times$ environment interactions}

Analyses of large-scale multi-environment trials (METs) have identified that large and regional GEIs can be a major impediment to genetic progress in breeding sunflowers in Argentina (de la Vega et al., 2001; de la Vega and Chapman, 2006). Repeatable GEIs may be exploited by selection for broad and/or specific adaptation, improving the overall efficiency of a breeding program, while unrepeatable GEIs must be treated as a source of experimental error. Predictable GEIs are those repeatable interactions determined by an environmental factor that can be predicted before planting, e.g., photoperiod, soil type and plant density. Since predictable GEIs are linked to location, planting date or management system, they can be exploited by grouping similar environments into mega-environments (ME; CIMMYT, 1989) and targeting specifically-adapted genotypes to each subdivision (Cooper and DeLacy, 1994).

Pattern analysis (i.e., joint use of classification and ordination; Cooper and DeLacy, 1994) has been recommended for the description of GEIs. This approach attempts to:

1. identify genotypes that have similar patterns of response across environments,

2. group environments that are similar in the way in which they discriminate among genotypes and

3. examine correlations between environments to exploit opportunities for indirect selection.

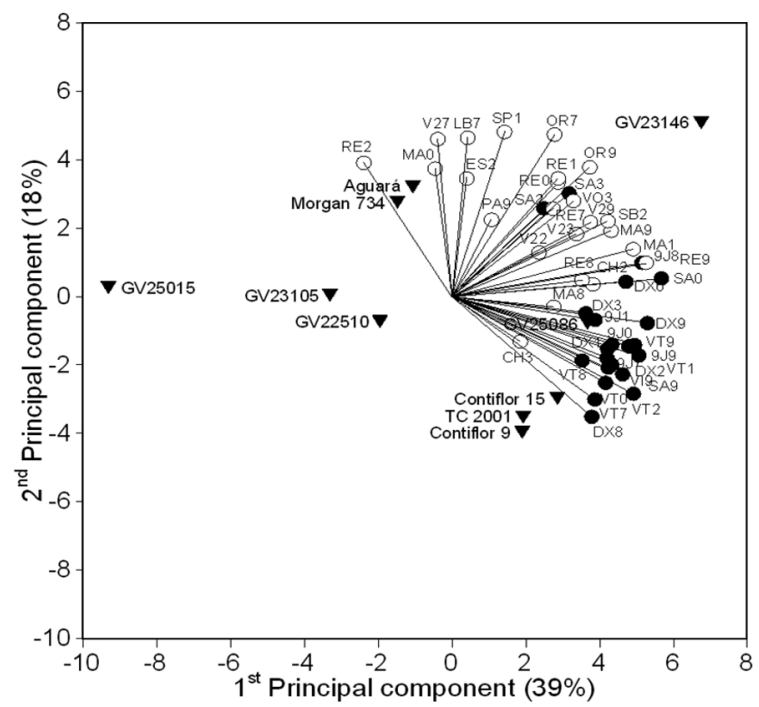

Figure 1: Biplot of the $1^{\text {st }}$ and the $2^{\text {nd }}$ principal components for oil yield of 10 sunflower hybrids grown in 46 trials. Genotypes are represented by points and trials by vectors. Solid and open circles identify central and northern environments, respectively (adapted from de la Vega and Chapman, 2006). 
Pattern analyses conducted on a dataset of 46 sunflower trials ( 7 years) where a reference set of 10 hybrids was tested (Figure 1; de la Vega and Chapman, 2006) showed that the central and northern sunflower regions of Argentina are orthogonal in terms of their average genotype discrimination effects for oil yield. The lack of overlap between both subregions indicates that these differences are repeatable and that subdivision and selection for specific adaptation to the identified MEs should be considered for increasing response to selection. However, according to the relative performance of broadly-adapted hybrids, it can be suggested that selection for broad adaptation to the undivided target region is possible and that both strategies must be compared in terms of predicted yield gains.

Correlations analyses suggested that photoperiod and minimum temperature are the main environmental factors underlying the observed GEIs, with the hybrids adapted to the northern $\mathrm{ME}$ improving their relative performance under relatively short photoperiods and high minimum temperatures, particularly when these conditions apply during the reproductive phenophases (de la Vega et al., 2001). Differences between central and northern subregions for these environmental factors during the different crop phases show a strong degree of repeatability (Chapman and de la Vega, 2002), which is consistent with the repeatability shown by the genotype-by-subregion interactions.

Apart from the strong differences observed between northern and central MEs in the manner in which they influence the relative performance of sunflower hybrids, large genotype $\times$ year (GYI), and genotype $\times$ location $\times$ year (GLYI) interactions for oil yield within regions were observed (Chapman and de la Vega, 2002; de la Vega and Chapman, 2006). For example, northern-adapted hybrids differing in maturity had contrasting responses to years (GYI interaction 3/4 as large as the G effect) in the northern environments (de la Vega and Chapman, 2006). It was suggested that the rainfall excesses during grain filling associated with the effect of the warm phase of El Niño Southern Oscillation (ENSO; e.g., 1997/98, 2002/03) could be determining an improvement in the relative performance of the early hybrids.

\section{Genetic progress}

The lack of genotypic association between central and northern MEs suggests that yield gains in northern environments would have been reduced if selection had been done in central environments and vice versa. Breeding company market experience suggests that the southern region constitutes a third ME. The capacity of pattern analysis to predict response to selection in highly heterogeneous TPEs and the market assumption about the southern region were tested through a retrospective analysis of genetic gain in the three subregions, using an 18-year MET dataset consisting of 324 advanced trials grown in 66 locations (de la Vega and Chapman, 2010). Mixed model and 0.90 quantile regressions analyses (Figure 2) indicated clear and continuous improvements in sunflower oil yield due to plant breeding during 25 years in the three MEs, with this progress having partially counteracted 
the decline in 'agronomic quality' of the sunflower growing environments, as the crop has been pushed toward more marginal, lower rainfall areas by the expansion of soybean production (de la Vega et al., 2007a). Moreover, this study confirmed the pattern-analysis-based prediction in showing lack of oil yield gains under northern and southern MEs from selection done in the central subregion (Figure 2).

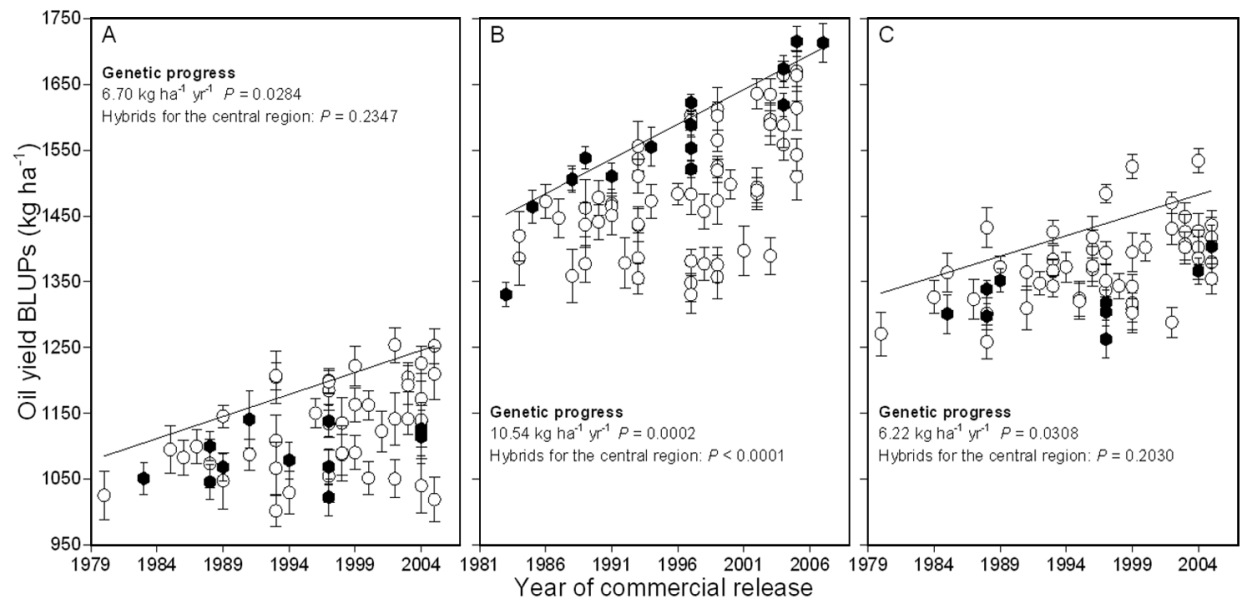

Figure 2: Best linear unbiased predictors (BLUPs) for oil yield for commercial sunflower hybrids in the northern (A), central (B) and southern (C) subregions of Argentina plotted against year of registration. Solid lines are 0.90 quantile regression functions. Solid symbols represent the hybrids developed by Advanta Semillas for the central ME (adapted from de la Vega and Chapman, 2010).

The central ME showed higher and more consistent genetic progress for oil yield than the other two, which is supported by the facts that this subregion is where most sunflower breeding programs of Argentina are situated and the one that shows both the lowest GEI to G ratio and the lowest within-trial heterogeneity (de la Vega and Chapman, 2010). Considering that the expansion of soybean production has reduced the relative size of the central subregion in favor of the other two MEs, the breeding and testing strategies followed by the sunflower breeding programs of Argentina should be revised with the aim of addressing this change in the relative importance of each sunflower growing subregion and thereby accelerating the genetic progress in the northern and southern MEs.

Biplot presentations of two-way (hybrids $\times$ traits) analyses (data not shown) summarized the results of 25 years of hybrid releases and showed that breeding has progressed to merge the best combinations of two original hybrid groups, i.e.:

1. intermediate-late to late maturity hybrids of white-striped seed, low grainoil concentration and high relative grain yield, mostly developed from locally-bred open pollinated varieties, and 
2. intermediate-early to early maturity hybrids of black seed, high grain-oil concentration and low relative grain yield, largely derived from Eastern European and US germplasm.

The breeding process towards this goal involved the selection of the maturities that tended to maximize yield potential and stability in each environment type. This process reduced the original range of phenological responses within subregions, but tended to concentrate on different maturity types among subregions (de la Vega et al., 2007b; de la Vega and Chapman, 2010). This observation confirmed that the three subregions are different MEs and that subdivision could result in a faster genetic progress, but also in a more diverse crop agro-ecological system, due to the exploitation of a broader range of genetic variability in breeding.

\section{Optimal selection strategies}

Conversion of GLI into G effect that can contribute to selection response is the main reason for the subdivision of large TPEs into MEs. If genotype $\times$ subregion interaction is large in comparison to $G$ effect, subdivision will be effective in increasing the $\mathrm{G}$ variance in the subregions relative to the original undivided area. However, division of testing resources could also result in loss of precision, i.e., reduced heritability $(H)$, in the prediction of genotype effects within the smaller subregions. Atlin et al. (2000) adapted Falconer's model of correlated response to selection to compare the relative effectiveness of the two strategies, which depends on the genetic correlation between the subregion and the undivided target region $\left(r_{g}\right)$ and the broad-sense $H$ (i.e., repeatability) estimated for each of both testing models. Subdivision of the central-north region of Argentina into central and northern MEs strongly increased the ratio of within-subregion $G$ to total GEI variance components. The equal division of the testing resources does not result in loss of precision in the prediction of the within-subregion $G$ effects relative to the prediction for the undivided target region. This, together with a low $r_{g}$ between the target region and the subregions, results in low correlated to direct response to selection ratios, which support the notion that dividing the sunflower central-north region of Argentina into central and northern MEs and exploiting specific adaptation would result in increased response to selection (de la Vega and Chapman, 2006).

Low ratios of within-subregion GLI to G discourage further subdivision. Mixed model analysis revealed that the highest portion of the total GEI within MEs accounts for in the GLYI. The variable and unpredictable nature of these interactions must be addressed by selecting for broad adaptation, ensuring that testing regimes adequately sample each crop target ME (Figure 3). The larger ratios of GLYI and GYI to G and the lower trial G variance components observed in the northern ME determine that to achieve $H$ s of more than 0.80 , trials would need to be conducted over at least four years. Conversely, in the central ME there is scope to redefine testing strategies by replacing years with locations at no costs in ability to predict genotype performance, resulting in the acceleration of genetic progress. 
These differences can be observed in Figure 1, where environments within the central subregion tended to be closer to each other, and northern environments were more divergent, indicating larger GEIs within this subregion.

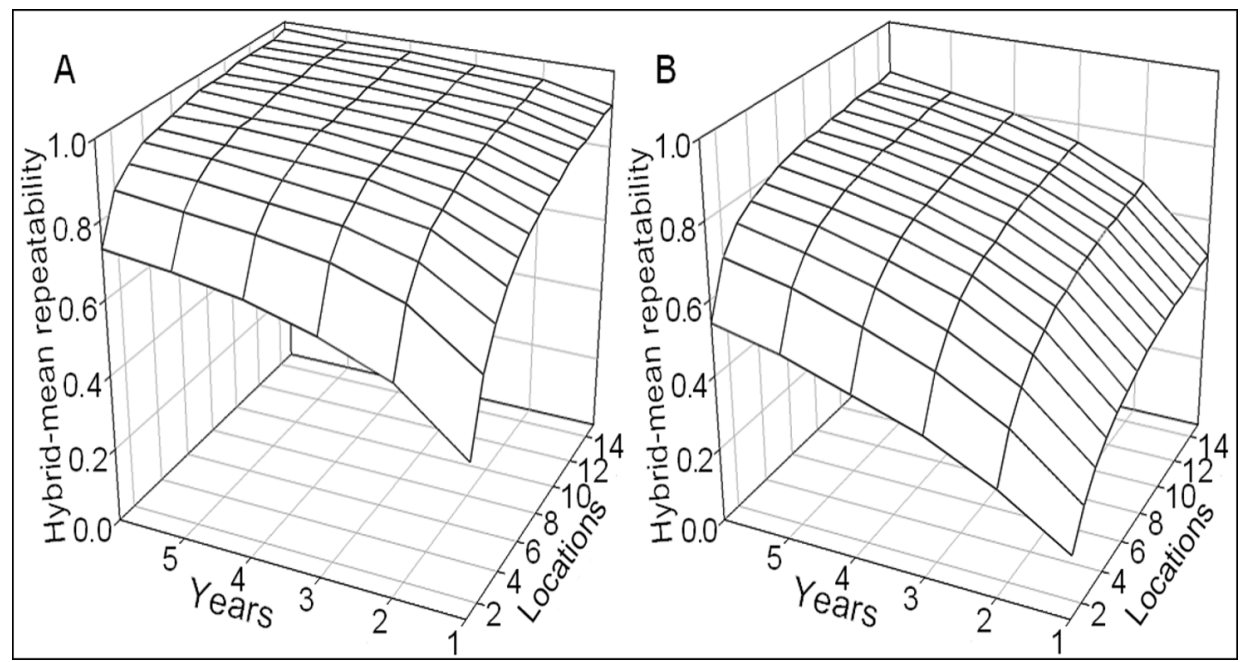

Figure 3: Surface plots of predicted hybrid-mean repeatability for oil yield in the central (A) and northern (B) MEs as the number of locations and years of testing in multienvironment trials are changed (assumes three replicates for each location-year combination), based on within-subregion components of variance (adapted from de la Vega and Chapman, 2006).

\section{Indirect selection criteria}

Most sunflower breeding stations in Argentina are located in the central ME. The value of the seed market of the northern $\mathrm{ME}$ is large enough to develop specifically adapted hybrids, but does not justify the establishment of a breeding station in situ. The use of managed-environments is an attempt to establish a selection regime, which is repeatable over years and provides discrimination among genotypes relevant to the target production system (Cooper et al., 1995). When normal October planting dates in a central location (Venado Tuerto) were delayed to December (V27, V29, V22, V23; Figure 1), they associated positively with the northern environments in the way in which they influenced the hybrid relative performance. This finding revealed the opportunity to use December plantings in Venado Tuerto as managed-environment for indirect selection for the northern ME (de la Vega et al., 2001; de la Vega and Chapman, 2010). When photoperiod was artificially extended to $15.5 \mathrm{~h}$ in this environment, genotypes reverted to responses similar to those of the normal planting date central environments, suggesting that photoperiod could be the environmental factor that is driving the association between the December plantings in central environments and the northern ME (de la Vega et al., 2001). 
Better knowledge of the physiological basis for the differential responses of genotypes to specific environments should improve the efficiency with which breeders characterize their germplasm in terms of G and GEI effects for yield, and hence increase the speed at which superior genotypes can be identified (i.e., ideotypebased selection). Two models of oil yield determination were used to study the physiological bases of the genotype $\times$ planting date interaction (GPI; i.e., October vs. December) effects observed in Venado Tuerto and to identify traits associated with specific adaptation to late plantings and the northern ME (de la Vega and Hall, 2002a and 2002b). These studies served to establish the importance of attributes and processes affecting crop performance in the post-anthesis phase (i.e., biomass accumulation during grain filling and changes in harvest index) in determining the observed GPI. Variations in grain set were the dominant determinant of the GPI for grain number and duration of grain filling was the main determinant of the GPI effects for grain weight and grain oil concentration. The diameter of the empty center and canopy stay green proved to be efficient easy-to-assess putative secondary indicators related to seed set and duration of grain filling, respectively - indicators that can be used to improve the efficiency of selection for specific adaptation to late plantings and the northern ME.

\section{CONCLUSIONS}

The design of breeding strategies aimed at increasing the current rates of yield progress in relevant TPEs requires the evaluation of the impact of GEI on realizable genetic gain. There are very few examples of production systems where a biological and statistical description of the GEIs has contributed to this goal like the one discussed in this review (e.g., Cooper, 1999) and no other in sunflower. In Argentina, adaptation analyses allowed to identify MEs, identify environmental factors driving GEIs, estimate the effect of differences in genotypic discrimination on genetic gain, assess the convenience of subdivision and selection for specific adaptation, design selection strategies and identify criteria for indirect selection. Further sunflower adaptation analyses in Argentina should concentrate on expanding these studies to the southern subregion, understanding the impact of the fungal diseases on GEIs, evaluating the impact of ENSO on the GYIs, determining some genetic bases of the observed GEIs and investigating the association between the Argentine sunflower growing regions and other sunflower growing countries in terms of genotypic discrimination to exploit opportunities for indirect selection. Adaptation analysis can also contribute to the investigation of strategies to improve the efficiency of breeding programs operating in other large and relevant sunflower production systems, like the EU, Russia and Ukraine. Moreover, the observed differences in genetic progress between MEs emphasize the need of developing adequate indirect selection strategies for all those secondary-target sunflower markets where local breeding is not conducted and only variable testing efforts are received. 


\section{ACKNOWLEDGEMENTS}

The sunflower studies cited in this review were supported by Advanta Semillas S.A.I.C. The mentoring and collaboration of Drs. Antonio J. Hall and Scott C. Chapman are gratefully acknowledged.

\section{REFERENCES}

Atlin, G.N., Baker, R.J., McRae, K.B., and Lu, X., 2000. Selection response in subdivided target regions. Crop Sci. 40: 7-13.

Chapman, S.C., and de la Vega, A.J., 2002. Spatial and seasonal effects confounding interpretation of sunflower yields in Argentina. Field Crops Res. 73: 107-120.

CIMMYT, 1989. An account of how priorities are set among mega-environments from a breeding perspective. Internal Document Number 17. Mexico DF.

Comstock, R.E., 1977. Quantitative genetics and the design of breeding programs. In: Proceedings of the International Conference on Quantitative Genetics, August 16-21, 1976. Iowa State University Press, Ames, USA, pp. 705-718.

Cooper, M., 1999. Concepts and strategies for plant adaptation research in rainfed lowland rice. Field Crops Res. 64: 13-34.

Cooper, M., and DeLacy, I.H., 1994. Relationships among analytical methods used to study genotypic variation and genotype-by-environment interaction in plant breeding multienvironment experiments. Theor. Appl. Genet. 88: 561-572.

Cooper, M., Woodruff, D.R., Eisemann, R.L., Brennan, P.S. and DeLacy, I.H., 1995. A selection strategy to accommodate genotype-by-environment interaction for grain yield of wheat: managed-environments for selection among genotypes. Theor. Appl. Genet. 90: 492-502.

de la Vega, A.J., and Chapman, S.C., 2006. Defining sunflower selection strategies for a highly heterogeneous target population of environments. Crop Sci. 46: 136-144.

de la Vega, A.J., and Chapman, S.C., 2010. Mega-environment differences affecting genetic progress for yield and relative value of component traits. Crop Sci. 50: 574-583.

de la Vega, A.J., and Hall, A.J., 2002a. Effects of planting date, genotype and their interactions on sunflower yield. II. Components of oil yield. Crop Sci. 42: 1202-1210.

de la Vega, A.J., and Hall, A.J., 2002b. Effects of planting date, genotype and their interactions on sunflower yield. I. Determinants of oil-corrected grain yield. Crop Sci. 42: 1191-1201.

de la Vega, A.J., Chapman, S.C., and Hall, A.J., 2001. Genotype by environment interaction and indirect selection for yield in sunflower. I. Two-mode pattern analysis of oil and biomass yield across environments in Argentina. Field Crops Res. 72: 17-38.

de la Vega, A.J., DeLacy, I.H. and Chapman, S.C., 2007a. Changes in agronomic traits of sunflower hybrids over 20 years of breeding in central Argentina. Field Crops Res. 100: 73-81.

de la Vega, A.J., DeLacy, I.H. and Chapman, S.C., 2007b. Progress over 20 years of sunflower breeding in central Argentina. Field Crops Res. 100: 61-72. 
